\title{
Methylation pattern analysis in prostate cancer tissue: identification of biomarkers using an MS-MLPA approach
}

\author{
Giorgia Gurioli ${ }^{1 \dagger}$, Samanta Salvi ${ }^{1 \dagger}$, Filippo Martignano ${ }^{1}$, Flavia Foca ${ }^{2}$, Roberta Gunelli ${ }^{3}$, Matteo Costantini ${ }^{4}$, \\ Giacomo Cicchetti ${ }^{5}$, Ugo De Giorgi ${ }^{6}$, Persio Dello Sbarba ${ }^{7}$, Daniele Calistri ${ }^{1}$ and Valentina Casadio ${ }^{1 *}$
}

\begin{abstract}
Background: Epigenetic silencing mediated by CpG island methylation is a common feature of many cancers. Characterizing aberrant DNA methylation changes associated with prostate carcinogenesis could potentially identify a tumour-specific methylation pattern, facilitating the early diagnosis of prostate cancer. The objective of the study was to assess the methylation status of 40 tumour suppressor genes in prostate cancer and healthy prostatic tissues.

Methods: We used methylation specific-multiplex ligation probe amplification (MS-MLPA) assay in two independent case series (training and validation set). The training set comprised samples of prostate cancer tissue $(n=40)$, healthy prostatic tissue adjacent to the tumor $(n=26)$, and healthy non prostatic tissue $(n=23)$, for a total of 89 DNA samples; the validation set was composed of 40 prostate cancer tissue samples and their adjacent healthy prostatic tissue, for a total of 80 DNA samples. Methylation specific-polymerase chain reaction (MSP) was used to confirm the results obtained in the validation set.

Results: We identified five highly methylated genes in prostate cancer: GSTP1, RARB, RASSF1, SCGB3A1, CCND2 $(P<0.0001)$, with an area under the ROC curve varying between 0.89 (95\% Cl 0.82-0.97) and $0.95(95 \% \mathrm{Cl} 0.90-1.00)$. Diagnostic accuracy ranged from $80 \%(95 \% \mathrm{Cl} 70-88)$ to $90 \%$ (95\% Cl 81-96). Moreover, a concordance rate ranging from $83 \%$ (95 \% Cl 72-90) to $89 \%$ (95 \% Cl 80-95) was observed between MS-MLPA and MSP.
\end{abstract}

Conclusions: Our preliminary results highlighted that hypermethylation of GSTP1, RARB, RASSF1, SCGB3A1 and CCND2 was highly tumour-specific in prostate cancer tissue.

Keywords: Methylation pattern, Prostate cancer, MS-MLPA, Early diagnosis

\section{Background}

Prostate cancer $(\mathrm{PCa})$ is the second most common malignancy in men [1]. Current diagnostic methods have increased the number of patients diagnosed with early $\mathrm{PCa}$, with a consequent benefit in survival, but have also led to overtreatment, reducing the cost-effectiveness of treatment $[2,3]$. Low sensitivity and specificity of the prostate-specific antigen (PSA) test, along with falsepositive results and unnecessary biopsies for PSA values

\footnotetext{
*Correspondence: valentina.casadio@irst.emr.it

${ }^{\dagger}$ Giorgia Gurioli and Samanta Salvi contributed equally to this work

1 Biosciences Laboratory, Istituto Scientifico Romagnolo per lo Studio e la Cura dei Tumori (IRST) IRCCS, Via P. Maroncelli 40, 47014 Meldola, Italy Full list of author information is available at the end of the article
}

between 4 and $10 \mathrm{ng} / \mathrm{ml}$, have created an urgent need for new biomarkers for PCa diagnosis $[4,5]$. New biomarkers for PCa diagnosis have been studied with the purpose of improving sensitivity and specificity of PSA; some of these are FDA-approved, e.g. PCA3 testing in urine [6], but none has been implemented into clinical practice. For this reason, new, robust markers to accurately characterize PCa must be sought.

It is well known that epigenetic modifications such as DNA methylation in CpG islands are correlated with cancer development, indicating that such events could represent early phenomena of carcinogenesis [7]. For this reason, DNA methylation could be a potential biomarker for the early diagnosis of PCa. Tumour suppressor genes 
are sometimes silenced by methylation and contribute to carcinogenesis, influencing mechanisms such as DNA repair and apoptosis [8]. Moreover, every tumour type has a specific methylation pattern which, when compared with corresponding healthy tissue, could be useful for diagnosis [9]. Several studies have focused on the relationship between modifications of epigenetic mechanisms and prostate carcinogenesis [10].

In the present study we chose to analyze the methylation status of a panel of 40 tumour suppressor genes (TIMP3, APC, CDKN2A, MLH1, ATM, RARB, CDKND2B, HIC1, CHFR, BRCA1, CASP8, CDKN1B, PTEN, BRCA2, CD44, RASSF1, DAPK1, VHL, ESR1, TP73, FHIT, CADM1, CDH13, GSTP1, CCND2, SCGB3A1, BNIP3, DLC1, HLTF, SFRP5, H2AFX, CACNA1AG, SFRP4, TWIST1, BCL2, CACNA1A, ID4, RUNX3, PRDM2 and TGIF) in PCa and healthy prostatic tissue to identify a tumour-specific methylation pattern that could facilitate early diagnosis. We chose this 40 genes panel because some of the selected genes (GSTP1, TIMP3, RARB, CDKN2A, HIC, APC, CD44, RASSF1, CDH13, DAPK, BCL2, SFRP5, RUNX3) are already known to be frequently methylated in $\mathrm{PCa}[11$, 12]: GSTP1 is the most widely studied and it is methylated in $70-80 \%$ of cases [9]. In addiction other genes, e.g. BRCA1, BRCA2, PTEN, TWIST1, are deregulated in vitro and in vivo due to their low expression or other genomic characteristics $[13,14]$. The remaining genes have important functions in different cancer-related processes, e.g. regulation of tumor growth, cell cycle control, differentiation and proliferation, cell adhesion and DNA damage repair.

We used the relatively new methodology of methylation specific-multiplex ligation dependent probe amplification (MS-MLPA) to evaluate epigenetic gene profiles in two independent cohorts of samples. This approach permits methylation analysis of multiple targets in a single experiment [15] and has been successfully used to evaluate the diagnostic or prognostic relevance of different markers in several tumor types including lung [16], rectal [17], breast [18] and bladder cancer [19, 20].

\section{Methods}

\section{Case series}

We analysed two independent case series: a training and validation set. For the training set, tissue samples were collected from patients submitted to prostatectomy between 2008 and 2011. Specifically, we collected 40 samples of paraffin-embedded PCa tissue, 26 of healthy prostatic tissue adjacent to the tumour, and 23 of healthy non prostatic tissue (seminal vesicles and bladder neck). In the training set we chose random samples, a number of which were not paired between cancer and healthy prostatic tissue. All paired cancer/healthy samples for the validation set were collected from patients who underwent prostatectomy in 2013. Specifically, we analysed 40 prostate cancer tissue samples and their adjacent healthy prostatic tissue, for a total of 80 samples.

All patients gave written informed consent to take part in the study, which was reviewed and approved by the local Ethics Committee ("Comitato Etico Area Vasta Romagna e IRST"). All samples were retrieved from the Archives of the Pathology Unit of the Morgagni-Pierantoni Hospital in Forlì.

\section{Macrodissection and DNA isolation}

Cancer and healthy tissue was selected and macrodissected on the basis of hematoxylin-eosin sections. Healthy prostatic tissue was macrodissected at a distance of $7 \mathrm{~mm}$ from the tumour sample. DNA was extracted using QIAamp DNA FFPE Tissue (Qiagen, Milan, Italy), according to the manufacturer's instructions, and quantified by spectrophotometry (NanoDrop ND-1000, Celbio, Milan, Italy). DNA from LNCaP cell line and peripheral blood of a healthy volunteer was extracted using QIAamp DNA Minikit (Qiagen), according to the manufacturer's instructions.

\section{MS-MLPA}

Methylation specific-multiplex ligation dependent probe amplification (MS-MLPA) was performed using at least $50 \mathrm{ng}$ of DNA dissolved in $1 \times$ TE buffer (Promega, Madison, WI, USA). DNA isolated from LNCaP cell line was used as internal control for MS-MLPA analysis. The methylation status of 40 tumor suppressor gene promoters was analysed using the ME001-C1 and ME003A1 kits (MRC-Holland, Amsterdam, The Netherlands) (Additional file 1: Table S1).

Two different probes that recognize two different sites of the promoter region were used for RASSF1, MLH1, SCGB3A1, CCND2, ID4, RARB, SFRP4, DLC1, H2AFX and HLTF genes. We considered the median value of the results of the two probes, assigning only one methylation value for each gene. $C D K N 2 B$ gene was excluded from the analysis because its probe is sensitive to improper HhaI digestion in FFPE samples.

MS-MLPA analysis was performed following the manufacturer's instructions. In brief, DNA was denatured and hybridization was performed by incubation at $60{ }^{\circ} \mathrm{C}$ for 16-18 h. Ligation and digestion reactions were then performed and samples were amplified by PCR. Digested probes could not be amplified exponentially during PCR and thus did not produce an amplification product. In contrast, if the DNA sample was methylated, DNA-probe hybrids were protected against $H$ haI enzyme digestion and the ligated probes generated an amplification product. 
Amplification products were analysed by ABI-3130 genetic Analyzer (Applied Biosystem, Foster City, CA). Electropherograms obtained were evaluated using Gene Mapper software (Applied Biosystem) and the peak areas of each probe were exported to a homemade excel spreadsheet.

In accordance with the manufacturer's instructions, we carried out "intrasample data normalization" by dividing the signal of each probe by the signal of each reference probe in the sample, thus creating as many ratios per probe as there were reference probes. We then calculated the median value of all probe ratios per probe, obtaining the normalization constant (NC). Finally, the methylation status of each probe was calculated by dividing the NC of a probe in the digested sample by the $\mathrm{NC}$ of the same probe in the undigested sample, and by multiplying this ratio by 100 to have a percentage value, as follows:

$$
\frac{\text { NC digested sample }}{\text { NC undigested sample }} \times 100
$$

We performed MS-MLPA analysis on four samples of peripheral blood of healthy volunteers, as controls for our analysis, finding absence of hypermethylation for all genes.

MS-MLPA reproducibility was assessed by performing three independent methylation profile analyses on LNCaP cell line. The methylation level for each gene was found to be the same in each experiment.

\section{MSP}

We used MSP as a confirmatory methodology to analyse the promoter methylation of five genes: GSTP1, RARB, RASSF1A, SCGB3A1 and CCND2. DNA was converted with sodium bisulphite using EZ DNA MethylationGold $^{\mathrm{TM}}$ kit (Zymo Research Corporation, Irvine, USA). The reactions were performed using $100 \mathrm{ng}$ of DNA extracted from LNCaP (methylated control) and from the peripheral blood of a healthy volunteer (unmethylated control), and 150 ng of FFPE DNA.

We performed real-time PCR using SYBR-GREEN master mix (Biorad, Milan, Italy) and primers specific for bisulphite-converted. Primer sequences for Actin $B$ were as follows: forward $5^{\prime}$-TGGTGATGGAGGAGGTTTAGT AAGT-3', reverse 5' ${ }^{\prime}$-AACCAATAAAACCTACTCCTCC CTTAA- ${ }^{\prime}$, as described elsewhere [21]. Real-time PCR was performed using Rotor Gene 3000 (Diatech pharmacogenetics, Jesi, Italy) under the following conditions: $95^{\circ} \mathrm{C}$ for $5 \mathrm{~min}$, then 40 cycles of $94{ }^{\circ} \mathrm{C}$ for $30 \mathrm{~s}, 62{ }^{\circ} \mathrm{C}$ for $60 \mathrm{~s}$ and $72{ }^{\circ} \mathrm{C}$ for $60 \mathrm{~s}$. We then evaluated PCR product specificity with melt curve analysis and set the $\mathrm{Ct}$ threshold at 0.02. For the subsequent preamplification PCR, $10 \mu \mathrm{l}$ of converted DNA was used when the Ct average value was $\geq 28$, or $6 \mu$ l when $<28$.
We performed a two-step MSP for RARB, RASSF1A, $S C G B 3 A 1$ and $C C N D 2$ methylation analysis, as previously described by Zhu et al. [22]. First, we performed a multiplex PCR using $\mathrm{C} 1000^{\mathrm{TM}}$ Thermal Cycler (Biorad) containing 4 primer pairs (called 'outer') for each gene with AmpliTaq Gold PCR kit (Applied Biosystems) [22]. The outer primers targeted methylated and unmethylated sequences in the same loci to enhance the amount of the four specific fragments used as templates for the second real-time PCR. Primer sequences for RARB, RASSF1A, SCGB3A1 and CCND2 are described in Table 1. Realtime PCR was carried out using SYBR-GREEN master mix (Biorad) and specific primers for methylated and unmethylated sequences (Table 1) for each gene. The previous preamplification products were diluted 1 to 10,000 for RARB, RASSF1A and SCGB3A1 and 1-5000 for $C C N D 2$. The reaction was performed under the following conditions: $95^{\circ} \mathrm{C}$ for $5 \mathrm{~min}$, then 40 cycles of $94{ }^{\circ} \mathrm{C}$ for $30 \mathrm{~s}, 58^{\circ} \mathrm{C}$ for $60 \mathrm{~s}$, and $72{ }^{\circ} \mathrm{C}$ for $60 \mathrm{~s}$.

For GSTP1 we only performed real-time PCR on $2 \mu \mathrm{l}$ of bisulphite-converted DNA samples. Primers for methylated and unmethylated sequences are shown in Table 1. The reaction was performed under the following conditions: $95^{\circ} \mathrm{C}$ for $3 \mathrm{~min}, 40$ cycles of $94{ }^{\circ} \mathrm{C}$ for $30 \mathrm{~s}, 56^{\circ} \mathrm{C}$ for $60 \mathrm{~s}$ and $72^{\circ} \mathrm{C}$ for $60 \mathrm{~s}$.

\section{Statistical analysis}

The sample size was calculated on the basis of the Bittner formula [23]: assuming $\alpha=0.01, \beta=0.10$, a standard deviation of gene methylation intensity of measurements on the base-two logarithmic scale $=0.7$ and a 1.5 -fold difference between the two classes (normal and tumour tissue). We compared clinical-pathological features in the training and validation sets using a non parametric statistical test. A two-dimensional unsupervised hierarchical cluster analysis of the methylation profile was performed using Euclidean distance as similarity measure, and clusters were combined using Ward's method. Normality of data distribution was tested using the Shapiro-Wilk test. If data distribution was not normal $(P>0.05)$, a non-parametric statistical test was used.

The relationship between methylation value and different subgroups of patients was analysed using the non-parametric statistical test (Wilcoxon test). For PCa samples, the relationship between methylation value and clinical-pathological features of patients were analysed using a non-parametric statistical test (Spearman correlation for PSA levels considered as continuous variable and Wilcoxon for the other variables).

In validation set, the genes showing a significant $P$ value in the Wilcoxon test were used to analyse the most discriminant cut-off values between $\mathrm{PCa}$ and $\mathrm{P}$ using ROC curve analysis. The true positive rates (sensitivity) 
Table 1 Primer sequences

\begin{tabular}{|c|c|c|c|}
\hline Gene & Outer primer sequences & Methylated primer sequences & Unmethylated primer sequences \\
\hline GSTP1 & & $\begin{array}{l}\text { 5'-TATCGTGGTTTATTTTTTAGTTCGA-3' } \\
\text { 3'-ATAAAAAAATTCGAATCTCTCCGA-5' }\end{array}$ & $\begin{array}{l}\text { 5'-TATTGTGGTTTATTTTTTAGTTTGA-3' } \\
\text { 3'-ATAAAAAAATTCAAATCTCTCCAAA-5' }\end{array}$ \\
\hline RARB & $\begin{array}{l}\text { 5'-TATGCGAGTTGTTTGAGGATTGGGA-3' } \\
\text { 3'-AATAATCATTTACCATTTTCCAAACTTA-5' }\end{array}$ & $\begin{array}{l}\text { 5'-TGTGAGAACGCGAGCGATTC-3' } \\
\text { 3'-CGACCAATCCAACCGAAACGA-5' }\end{array}$ & $\begin{array}{l}\text { 5'-TTGGGATGTTGAGAATGTGAGTGATTT-3' } \\
3^{\prime} \text {-CTTACTCAACCAATCCAACCAAAACAA-5' }\end{array}$ \\
\hline RASSF1 & $\begin{array}{l}\text { 5'-GTTTAGTTTGGATTTTGGGGGAG-3' } \\
\text { 3'-CCCACAACTCAATAAACTCAATAAACTCAAACTC-5' }\end{array}$ & $\begin{array}{l}\text { 5'-GGGTTCGTTTTGTGGTTTCGTTC-3' } \\
\text { 3'-TAACCCGATTAAACCCGTACTTCG-5' }\end{array}$ & $\begin{array}{l}\text { 5'-GGGGTTTGTTTTGTGGTTTTGTTT-3' } \\
\text { 3'-AACATAACCCAATTAAACCCATACTTCA-5' }\end{array}$ \\
\hline SCGB3A1 & $\begin{array}{l}\text { 5'-AGTGAGGATATTTAGAGAAATTTAGG-3' } \\
\text { 3'-ATCCCTACCTCTAATCCCAA-5' }\end{array}$ & $\begin{array}{l}\text { 5'-GCGTCGAGGTTAGTTCG-3' } \\
\text { 3'-GTAAACGCCTTCTACGCCTAA-5' }\end{array}$ & $\begin{array}{l}\text { 5'-GTGTTGAGGTTAGTTTTGAAGA-3' } \\
\text { 3'-TAAACACCTTCTACACCTAAAA-5' }\end{array}$ \\
\hline CCND2 & $\begin{array}{l}\text { 5'-TATTTTTTGTAAAGATAGTTTTGATTTAAGG-3' } \\
\text { 3'-TTTCCCCGAAAACATAAAACCTCC-5' }^{\prime}\end{array}$ & $\begin{array}{l}\text { 5'-GGCGGATTTTATCGTAGTCG-3' } \\
3^{\prime} \text {-CTCCACGCTCGATCCTTCG-5' }\end{array}$ & $\begin{array}{l}\text { 5'-AGAGTATGTGTTAGGGTTGATT-3' } \\
3^{\prime} \text {-ACATCCTCACCAACCTCCA-5' }\end{array}$ \\
\hline
\end{tabular}

were plotted against the false positive rates (1-specificity) for all classification points. Cohen's kappa coefficient was calculated to measure the concordance rate of MS-MLPA and MSP methods. A kappa value of more than 0.60 was regarded as showing strong agreement.

A stepwise logistic regression model was used in the validation set to analyse the relative risks (RR) and their $95 \%$ CIs for patient status $(\mathrm{PCa} / \mathrm{P})$ and methylation status for selected genes.

All $P$ values reported were two-sided and evaluated at the 0.05 level. Correction for multiple testing was performed using the Benjamini-Hochberg approach. Cluster analysis was performed with $\mathrm{R}$ software (version 3.0.1). All other statistical analyses were performed with STATA/MP 10.1 for Windows (Stata Corp LP).

\section{Results}

The clinical-pathological characteristics are summarized in Table 2. We analysed two independent sets of samples: a training and a validation set. The sensitivity of the MSMLPA technique was evaluated by constructing a curve based on the generation of different proportions $(5,10$, $20,40,80,100 \%)$ of DNA derived from a prostate cancer cell line (LNCaP) with known methylation of GSTP1, RASSF1, SCGB3A1, CASP8, RARB, CD44, APC, RUNX3, $C C N D 2$, spiked in genomic DNA (control) from a blood sample of a healthy donor. We found good linearity and a positive correlation between methylated DNA input and MS-MLPA results (Fig. 1). We also showed that the MSMLPA technique is capable of detecting very low methylation percentages (at least $5 \%$ ).

\section{Training set}

The methylation status of 40 tumour suppressor genes was analysed in 89 samples. Unsupervised cluster analysis for methylation profile identified two principal groups: one composed mainly of PCa tissue, the other mainly by healthy prostatic tissue adjacent to the tumour (defined as 'P') and healthy non prostatic tissue ('C') (Fig. 2).
Table 2 Case series

\begin{tabular}{|c|c|c|c|}
\hline & $\begin{array}{l}\text { Training set } \\
n=40\end{array}$ & $\begin{array}{l}\text { Validation set } \\
n=40\end{array}$ & $P$ value ${ }^{a}$ \\
\hline \multicolumn{4}{|l|}{ Age, years } \\
\hline$\leq 70$ & 32 & 33 & 0.775 \\
\hline$>70$ & 8 & 7 & \\
\hline \multicolumn{4}{|l|}{ Gleason score } \\
\hline$\leq 6$ & 18 & 13 & 0.251 \\
\hline$>6$ & 22 & 27 & \\
\hline \multicolumn{4}{|l|}{ Pathological stage } \\
\hline T2a & 5 & 5 & \\
\hline $\mathrm{T} 2 \mathrm{~b}$ & 1 & 0 & \\
\hline $\mathrm{T} 2 \mathrm{C}$ & 17 & 16 & 0.554 \\
\hline T3a & 12 & 17 & \\
\hline $\mathrm{T} 3 \mathrm{~b}$ & 5 & 2 & \\
\hline Median PSA (range) & $6.77(3.19-33.14)$ & $5.81(2.65-24.00)$ & 0.1988 \\
\hline
\end{tabular}

a The two groups were equally distributed for age, Gleason score, pathological stage and PSA. The Chi square test was used for age and Gleason score to determine statistical differences between training and validation sets; the Fisher test was used for pathological stage and the Wilcoxon test for PSA value

The median methylation levels for each gene in the three groups of samples are shown in Additional file 2: Table S2. Considering healthy and control samples, we observed statistically different methylation status for only 2 genes: the median methylation status of CASP8 was 9.92 (range 0.00-28.90) in P vs. 0.00 (range 0.00-9.50) in $C(P<0.0001)$ and 9.45 (range $0.00-28.80)$ in $P$ vs. 0.00 (range $0.00-20.80)$ in C for SCGB3A1 $(P=0.0005)$ (Additional file 3: Figure S1). With the exception of these two genes, there were no differences in the methylation profile of the $\mathrm{P}$ and $\mathrm{C}$ samples. We compared the methylation status of the $\mathrm{PCa}$ and $\mathrm{P}$ groups to test the role of methylation in the early diagnosis of PCa. Comparative analysis showed that 12 genes had statistically higher methylation in tumour tissue (Table 3). No significant correlations were found between methylation status and clinical-pathological characteristics. 


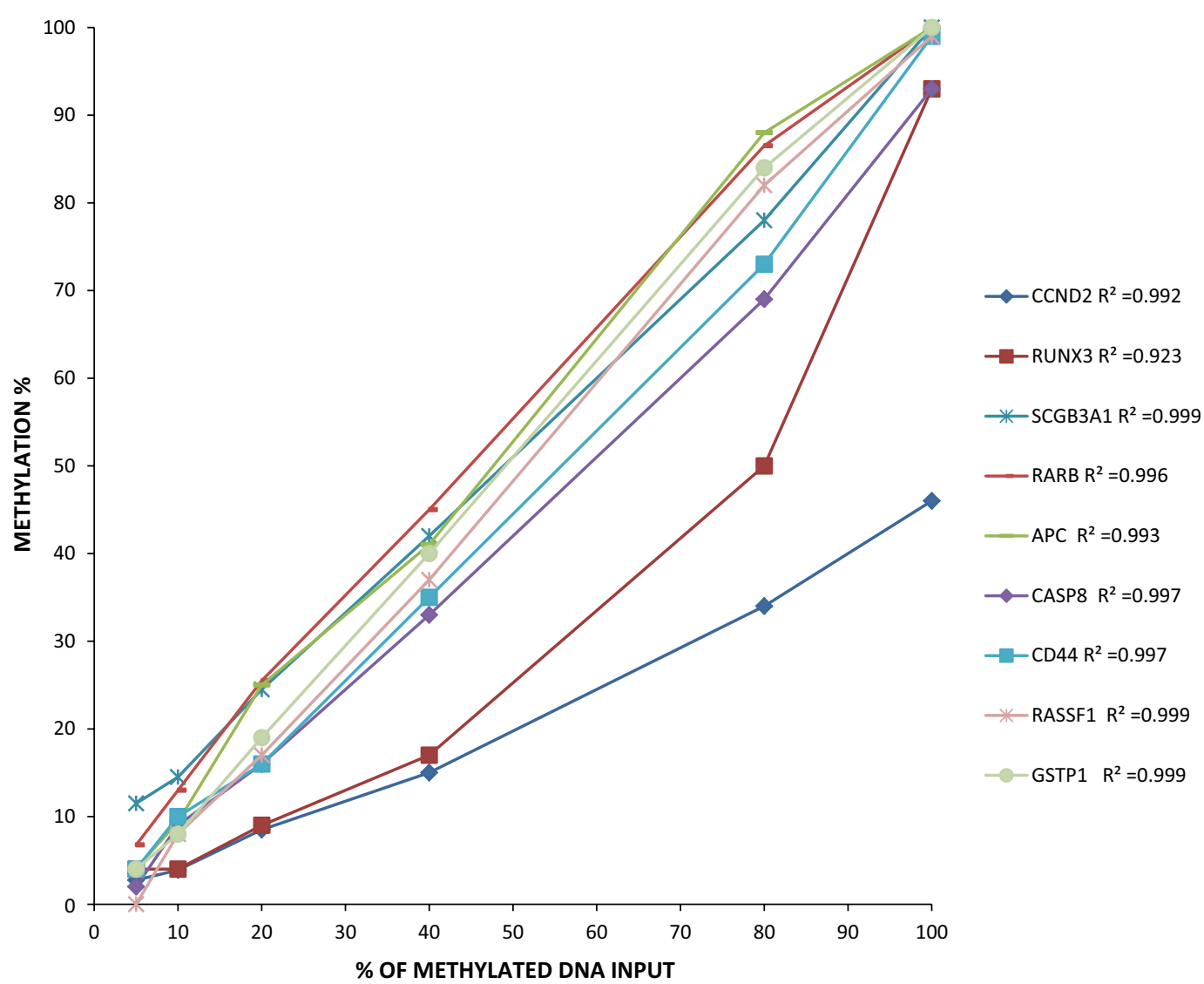

Fig. 1 Correlation between different percentages of methylated DNA input (LNCaP cell line, $X$ axis) and methylation percentage results obtained using the MS-MLPA technique ( $Y$ axis). Results for CCND2, RUNX3, SCGB3A1, RARB, APC, CASP8, CD44, RASSF1 and GSTP1 are reported with corresponding $R^{2}$ results

\section{Validation set}

Forty PCa tissue samples and $40 \mathrm{P}$ samples were evaluated for the same panel of tumour suppressor genes. As in the training set, we identified two cluster groups in validation set, one composed of PCa samples, the other of $\mathrm{P}$ samples, the latter showing a lower methylation profile (Fig. 3). The median methylation levels for each gene in the two groups of samples are shown in Additional file 2: Table S2. Comparative analysis confirmed that gene methylation status differed between tumour and healthy tissue in 9 out of 12 genes (Table 3). We then performed receiver operating characteristic (ROC) curve analyses for each of these genes (Fig. 4): five genes (GSTP1, RARB, RASSF1, SCGB3A1 and CCND2) were highly specific in discriminating between prostate cancer and adjacent normal tissue, with an area under ROC curve (AUC) ranging from 0.89 (95\% CI 0.82-0.97) to 0.95 (95\% CI 0.90-1.00). ROC curve analysis also identified the best methylation cut-off: we considered the promoters showing a ratio $\geq 0.20$ as methylated, while those with a ratio $<0.20$ were considered as unmethylated. The overall diagnostic accuracy for the five genes varied between 80 and $90 \%$ (Table 4). A stepwise regression analysis with a 0.20 probability removal was carried out in the validation set to evaluate the capability of GSTP1, RARB, RASSF1, $S C G B 3 A 1$ and CCND2 gene methylation to predict the risk of PCa. RARB and SCGB3A1 proved to be independent variables that indicated a relative risk of there being prostate cancer of 1.14 (95\% CI 0.99-1.31, $P=0.058$ ) and 1.10 (95\% CI 1.01-1.21, $P=0.028$ ), respectively.

Correlation analysis of methylation status and clinical-pathological characteristics showed that RUNX3 was significantly correlated with PSA levels: its methylation status was positively associated with PSA levels $(P=0.0001)$. We also performed methylation specific PCR (MSP) analysis for GSTP1, RARB, RASSF1, $S C G B 3 A 1$ and $C C N D 2$ to confirm their methylation status previously identified with the MS-MLPA analysis. We used Cohen's kappa coefficient to assess the concordance rate between MS-MLPA and MSP analyses. The kappa coefficient was greater than 0.6 for each gene, indicating strong agreement that ranged from 83 to $89 \%$. 


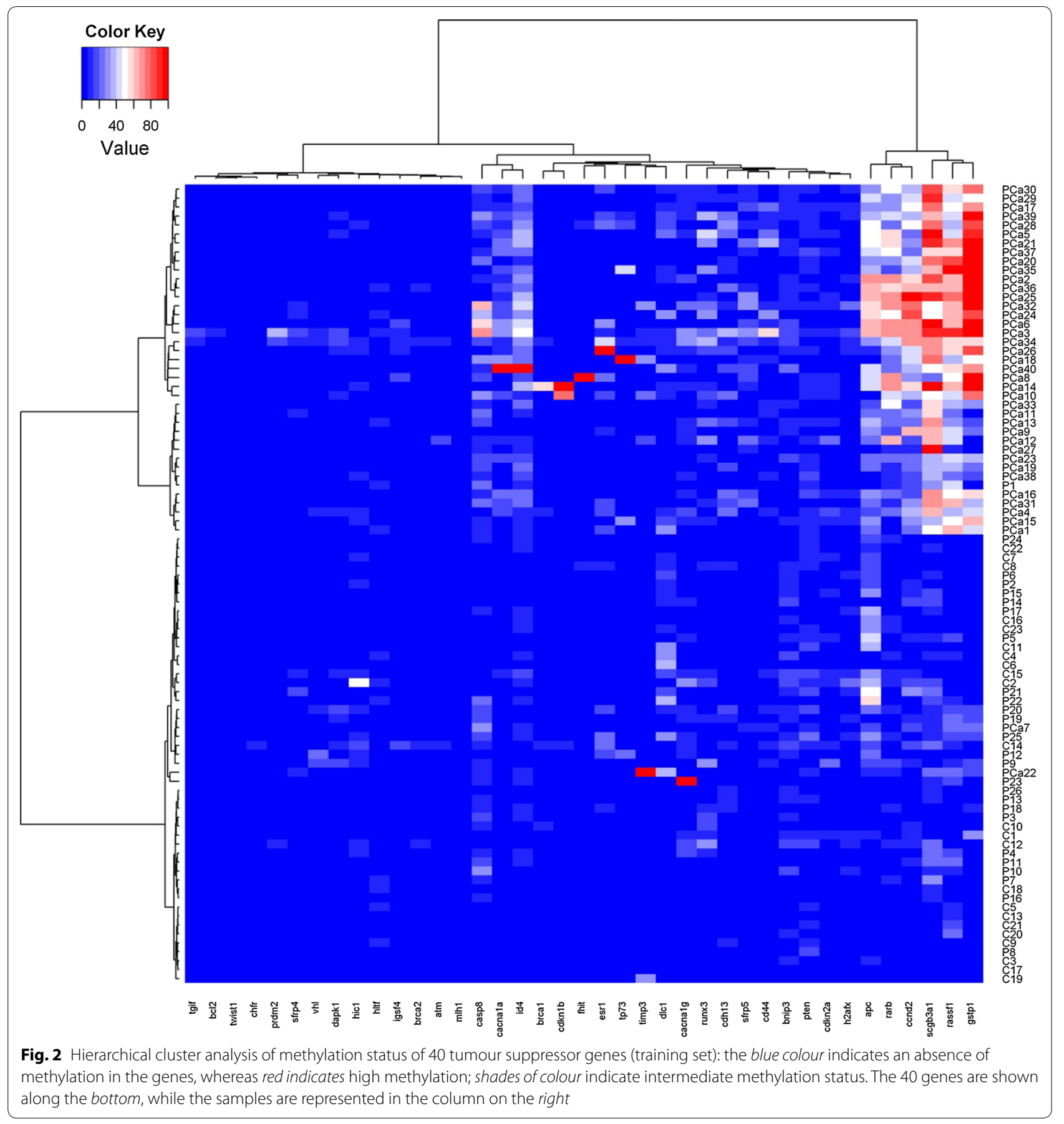

We also evaluated the global methylation status of 28 paired cancer and normal samples using ELISA assay (MethyFlash Methylated DNA Quantification Kit, Epigentek, NY, USA) and obtained a global methylation percentage. Nineteen $(68 \%)$ patients showed a $5-\mathrm{mC}$ (5-methylcytosine) \% decrease in cancer tissue compared to normal adjacent tissue (data not shown).
We compared early tumors of PCa (defined as Gleason score $\leq 6$ or T2 pathological stage) with normal prostate tissue (P), but we also compared all PCa with P. We identified the same statistically significant differences in the two comparisons except for these genes: CDH13, RUNX3 in training set and $H I C 1$ in validation set. For training set, CDH13 and RUNX3 had statistical significance in all PCa 
Table 3 Difference in methylated genes between cancer (PCa) samples and healthy (P) samples adjacent to the tumour

\begin{tabular}{|c|c|c|c|c|c|c|c|}
\hline \multirow[t]{3}{*}{ Gene } & \multicolumn{3}{|l|}{ Training set } & \multicolumn{3}{|l|}{ Validation set } & \multirow{3}{*}{$\begin{array}{l}\text { AUC Validation set } \\
(95 \% \mathrm{Cl})\end{array}$} \\
\hline & \multicolumn{2}{|c|}{ Median value (range) } & \multirow[t]{2}{*}{$P$ value* } & \multicolumn{2}{|c|}{ Median value (range) } & \multirow[t]{2}{*}{$P$ value* } & \\
\hline & $\mathrm{PCa}$ & $\mathbf{P}$ & & $\mathrm{PCa}$ & $\mathbf{P}$ & & \\
\hline GSTP1 & $69.70(0.00-100.00)$ & $0.00(0.00-18.20)$ & $<0.0001$ & $35.50(0.00-100.00)$ & $2.81(0.00-30.18)$ & $<0.0001$ & $0.89(0.82-0.97)$ \\
\hline RASSF1 & $49.25(11.10-100.00)$ & $5.00(0.00-45.60)$ & $<0.0001$ & 46.69 (9.88-79.98) & $9.63(0.00-74.81)$ & $<0.0001$ & $0.92(0.85-0.98)$ \\
\hline CCND2 & $35.10(4.40-94.60)$ & $5.75(0.00-27.10)$ & $<0.0001$ & $33.29(7.26-100.00)$ & $5.41(0.00-38.45)$ & $<0.0001$ & $0.92(0.86-0.98)$ \\
\hline SCGB3A1 & $65.90(12.40-100.00)$ & $9.45(0.00-28.80)$ & $<0.0001$ & $56.47(0.00-100.00)$ & $10.35(0.00-35.04)$ & $<0.0001$ & $0.95(0.90-1.00)$ \\
\hline$R A R B$ & $29.95(4.60-71.50)$ & $4.65(0.00-16.10)$ & $<0.0001$ & $35.89(2.29-100.00)$ & $4.55(0.00-18.71)$ & $<0.0001$ & $0.94(0.88-0.99)$ \\
\hline ID4 & $22.65(4.30-100.00)$ & $5.15(0.00-11.90)$ & $<0.0001$ & $14.41(0.00-45.32)$ & $5.08(0.00-16.08)$ & $<0.0001$ & $0.86(0.78-0.94)$ \\
\hline CACNA1A & $12.95(0.00-100.00)$ & $0.00(0.00-2.13)$ & 0.0001 & $6.32(0.00-68.89)$ & $0.00(0.00-14.94)$ & 0.0001 & $0.74(0.64-0.84)$ \\
\hline SFRP5 & $7.50(0.00-29.30)$ & $0.00(0.00-0.00)$ & $<0.0001$ & $8.35(0.00-39.01)$ & $0.00(0.00-10.79)$ & $<0.0001$ & $0.82(0.73-0.91)$ \\
\hline$A P C$ & $32.55(0.00-71.00)$ & $4.88(0.00-59.10)$ & 0.0001 & $36.77(0.00-91.80)$ & $5.89(0.00-38.90)$ & $<0.0001$ & $0.84(0.75-0.93)$ \\
\hline CD44 & $5.60(0.00-57.40)$ & $0.00(0.00-10.30)$ & 0.0009 & $5.92(0.00-59.33)$ & $4.08(0.00-29.70)$ & 0.1391 & - \\
\hline $\mathrm{CDH} 13$ & $0.00(0.00-39.00)$ & $0.00(0.00-14.40)$ & 0.0087 & $9.43(0.00-41.21)$ & $5.99(0.00-27.62)$ & 0.0557 & - \\
\hline RUNX3 & $3.75(0.00-44.10)$ & $0.00(0.00-29.60)$ & 0.0088 & $9.71(0.00-47.22)$ & $8.38(0.00-44.90)$ & 0.3351 & - \\
\hline
\end{tabular}

AUC area under ROC curve

* Wilcoxon test: prostate cancer samples (PCa) vs. healthy adjacent prostate samples (P)

vs. P comparison but not in early tumors. For the validation set, HIC1 acquired statistical significance in early tumors but this was not confirmed in all PCa series vs. P.

\section{Discussion}

Aberrant DNA methylation usually occurs at an early stage in cancer, rendering DNA methylation biomarkers good candidates for early cancer detection. In the present study we used an MS-MLPA approach to identify a panel of tumour suppressor genes differentially methylated in prostate cancer with respect to healthy tissue. The MSMLPA method is a highly sensitive method [17, 20, 24] that is capable of identifying several promoter regions using a small quantity of DNA [25, 26]. We detected 12 genes with high methylation levels in tumour tissue compared to healthy tissue in the training set, nine of which were subsequently confirmed in the validation set. Of these nine genes, five (GSTP1, RARB, RASSF1, SCGB3A1 and $C C N D 2$ ) discriminated between tumour and healthy tissue with a diagnostic accuracy of $\geq 80 \%$. As the MSMLPA technique is designed to assess the methylation status of single $\mathrm{CpG}$ dinucleotides, a negative finding at a single $\mathrm{CpG}$ dinucleotide is not sufficient to rule out methylation at a given island. For this reason we confirmed the methylation status of these five genes by MSP, a simpler and less expensive method, based on bisulphite DNA conversion.

The concordance rate between MS-MLPA (based on $H$ haI enzymatic cleavage of unmethylated sequences) and MSP (based on bisulphite conversion of unmethylated cytosines to uracils) revealed strong agreement between the two methods.

Although hypermethylation in the promoter regions of tumour suppressor genes is often observed in cancer, global DNA hypomethylation occurs in cancer tissue with respect to normal tissue $[27,28]$. In line with these literature data, we observed a lower $5-\mathrm{mC} \%$ in cancer compared to normal adjacent tissue in our small cohort of 28 patients (validation set) (data not shown).

Numerous studies investigating GSTP1, RARB and RASSF1 DNA promoter methylation have reported that hypermethylation in prostate tumour tissue may represent a biomarker of early cancer diagnosis, in accordance with our results $[29,30]$. There are few studies in literature on the hypermethylation of SCGB3A1 and CCND2 in prostate tumour tissue. SCGB3A1 is a growth-inhibitory cytokine which is downregulated in the majority of prostate, breast, lung, pancreatic and nasopharyngeal cancers due to DNA promoter hypermethylation [31]. CCND2 is a cell cycle-regulatory gene whose altered expression makes it function as an oncogene or tumor suppressor gene [32]. Padar et al. [33] observed an association between CCND2 inactivation and promoter region hypermethylation, reporting a positive correlation between methylation frequency and high Gleason score group suggestive of a prognostic role of the gene. Although SCGB3A1 and CCND2 methylation in prostate cancer has been reported, the possibility of using these markers for the early diagnosis of $\mathrm{PCa}$ has never been fully investigated. 


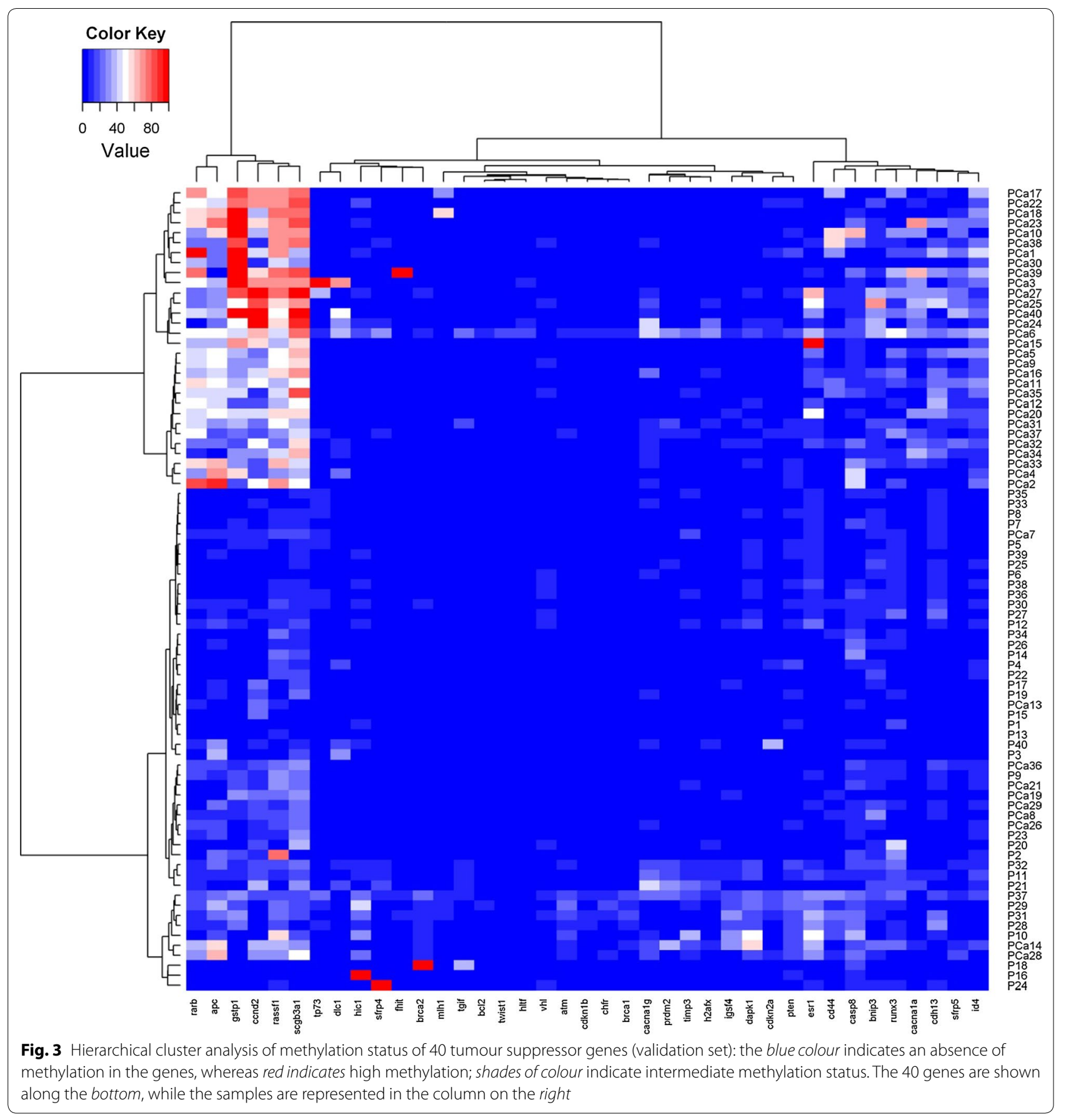

We also highlighted a similar methylation profile for healthy prostatic tissue adjacent to the tumour and healthy non prostatic tissue, with the exception of CASP8 and SCGB3A1, which showed a statistically higher methylation in the former.

The methylation level of SCGB3A1 gradually increased from low in healthy non prostatic tissue, to intermediate in healthy prostatic samples, and to high in PCa tissue, suggesting a role in early tumourigenesis. For this reason, we hypothesised that SCGB3A1 could be an important biomarker for cancer in non cancerous prostatic tissue. Noteworthy, RASSF1 and SCGB3A1 had similar sensitivity values both in early and in locally advanced tumors (Table 4), thus demonstrating they could be potentially 

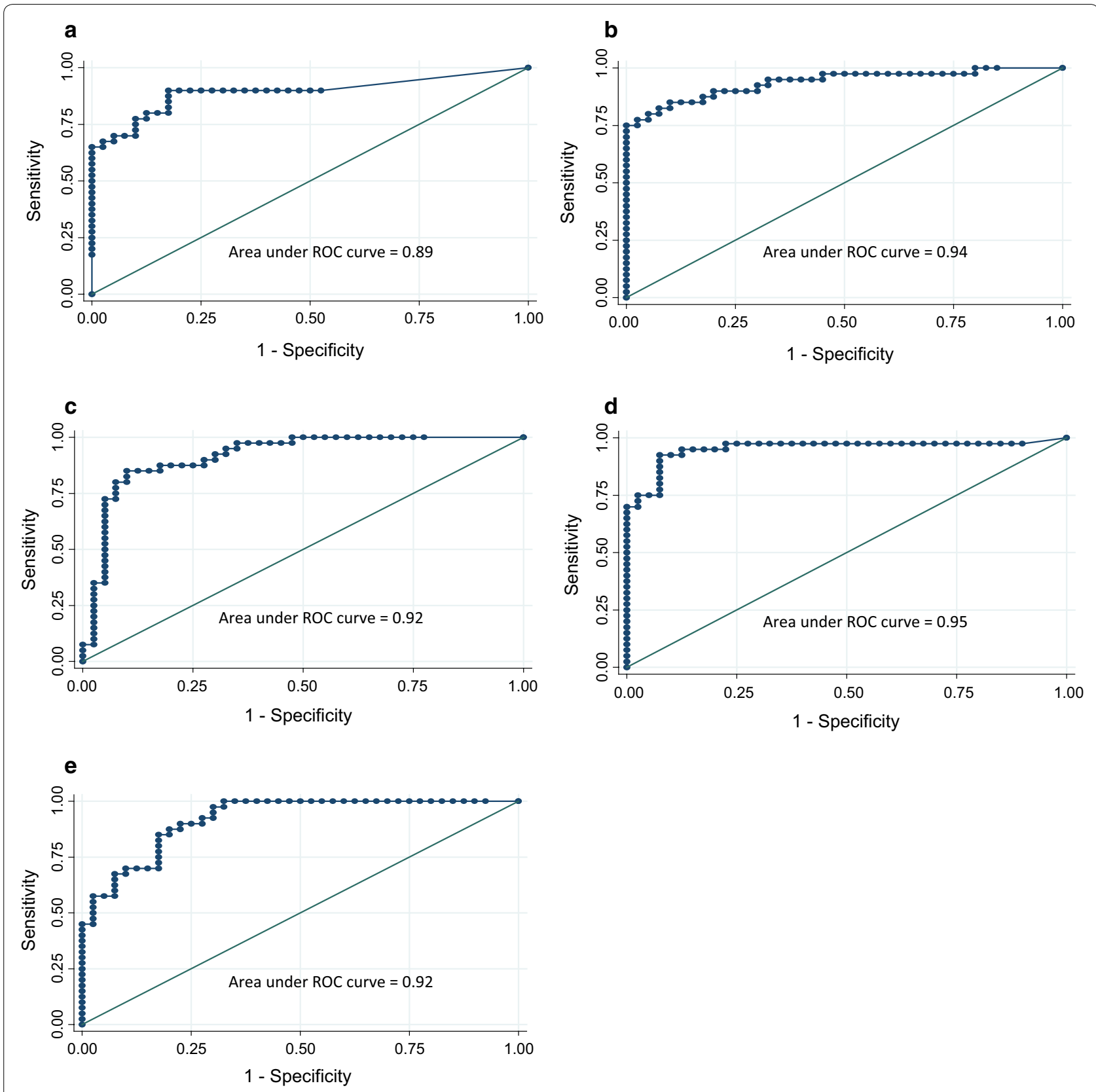

Fig. 4 ROC curve analysis of the five genes highly specific in discriminating prostate cancer from healthy tissue: a GSTP1, b RARB, c RASSF1, d SCGB3A1, and e CCND2

involved in the very earliest phases of carcinogenesis. This is an important characteristic for early diagnostic biomarkers.

In recent years, DNA promoter methylation has also been acknowledged as a biomarker to support clinical decision-making for suspected PCa. An important clinical problem is to determine which patients with a suspicion of $\mathrm{PCa}$ and initial negative biopsy should be referred for a second biopsy [34]. As some studies have recently suggested that false-negative first biopsies occur in around $20-25 \%$ of patients [35-37], it is essential to unmask the cases of PCa among the negative core biopsies, thus eliminating the need for a repeat biopsy. Two studies, the MATLOC study [35] and the more recent DOCUMENT study [36] have shown that three methylation markers (GSTP1, APC and RASSF1) are capable of identifying $\mathrm{PCa}$ among negative core biopsies. 
Table 4 Diagnostic accuracy

\begin{tabular}{|c|c|c|c|c|c|}
\hline Gene & $\begin{array}{l}\text { Overall sensitivity } \\
(n=40) \%\end{array}$ & $\begin{array}{l}\text { Early tumors sensitivity } \\
(\mathrm{n}=22) \%^{\mathrm{a}}\end{array}$ & $\begin{array}{l}\text { Locally advanced } \\
\text { tumors sensitivity } \\
(n=18) \%^{b}\end{array}$ & $\begin{array}{l}\text { Overall } \\
\text { specificity } \\
(n=40) \%\end{array}$ & $\begin{array}{l}\text { Overall } \\
\text { accuracy } \\
(n=80) \% \\
\end{array}$ \\
\hline \multicolumn{6}{|l|}{ RARB } \\
\hline Rate (95\% Cl) & $\begin{array}{l}30 / 40 \\
75(59-87)\end{array}$ & $\begin{array}{l}15 / 22 \\
68(49-87)\end{array}$ & $\begin{array}{l}15 / 18 \\
83(66-100)\end{array}$ & $\begin{array}{l}40 / 40 \\
100(91-100)\end{array}$ & $\begin{array}{l}70 / 80 \\
88(78-94)\end{array}$ \\
\hline \multicolumn{6}{|l|}{ GSTP1 } \\
\hline Rate $(95 \%$ Cl) & $\begin{array}{l}28 / 40 \\
70(53-83)\end{array}$ & $\begin{array}{l}14 / 22 \\
64(44-84)\end{array}$ & $\begin{array}{l}14 / 18 \\
78(59-97)\end{array}$ & $\begin{array}{l}37 / 40 \\
93(80-98)\end{array}$ & $\begin{array}{l}65 / 80 \\
81(71-89)\end{array}$ \\
\hline \multicolumn{6}{|l|}{ CCND2 } \\
\hline Rate (95 \% Cl) & $\begin{array}{l}27 / 40 \\
68(51-81)\end{array}$ & $\begin{array}{l}12 / 22 \\
55(34-76)\end{array}$ & $\begin{array}{l}15 / 18 \\
83(66-100)\end{array}$ & $\begin{array}{l}37 / 40 \\
93(80-98)\end{array}$ & $\begin{array}{l}64 / 80 \\
80(70-88)\end{array}$ \\
\hline \multicolumn{6}{|l|}{ RASSF1 } \\
\hline Rate (95 \% Cl) & $\begin{array}{l}35 / 40 \\
88(73-96)\end{array}$ & $\begin{array}{l}18 / 22 \\
82(66-98)\end{array}$ & $\begin{array}{l}17 / 18 \\
94(83-100)\end{array}$ & $\begin{array}{l}33 / 40 \\
83(67-93)\end{array}$ & $\begin{array}{l}68 / 80 \\
85(75-92)\end{array}$ \\
\hline \multicolumn{6}{|l|}{ SCGB3A1 } \\
\hline Rate $(95 \%$ Cl) & $\begin{array}{l}38 / 40 \\
95(83-99)\end{array}$ & $\begin{array}{l}21 / 22 \\
95(86-100)\end{array}$ & $\begin{array}{l}17 / 18 \\
94(83-100)\end{array}$ & $\begin{array}{l}34 / 40 \\
85 \text { (70-94) }\end{array}$ & $\begin{array}{l}72 / 80 \\
90(81-96)\end{array}$ \\
\hline
\end{tabular}

a Early tumors: $\mathrm{GS} \leq 6$ or T2

b Locally advanced tumors: GS $>6$ or T3

The role of GSTP1, RARB and RASSF1 DNA promoter methylation as a non invasive biomarker for the early diagnosis of PCa has been widely investigated in body fluids $[38,39]$ using the MSP technique. A number of authors have also suggested that urine cell-free DNA could represent a source of cost-effective and non invasive biomarkers [36, 40-42], e.g. specific promoter region methylation. To the best of our knowledge, SCGB3A1 and CCND2 have not been hypothesised as potential non invasive biomarkers for early diagnosis and it would thus be interesting to analyse them in urine or plasma cell-free DNA.

\section{Conclusions}

Our preliminary results, obtained in a single-institution study with limited enrolment, showed that the hypermethylation of GSTP1, RARB, RASSF1, SCGB3A1 and $C C N D 2$ was highly tumour-specific in prostate cancer tissue. We verified the potential of these markers in two independent case series and using two different techniques. Larger prospective studies are now needed to investigate these genes in body fluids.

\section{Additional files}

Additional file 1: Table S1. Function and localisation of tumor suppressor genes.

Additional file 2: Table S2. Median methylation values.

Additional file 3: Figure S1. Methylation percentages of the two genes (CASP8 and SCGB3A1) in P, C and PCa samples that were differentially methylated in $C$ and $P$ samples in the training set. The figure highlights a higher methylation percentage for PCa than for healthy samples, but also a higher methylation percentage for $P$ than for $C$ samples in both genes.

\section{Authors' contributions}

GG carried out the molecular experiments and drafted the manuscript. SS carried out the molecular experiments and drafted the manuscript. FM carried out the molecular experiments. FF performed the statistical analysis. RG was responsible for patients recruitment. MC contributes to clinical data regarding patients. GC was responsible for patients recruitment. UDG contributes to clinical data regarding patients. PDS participated in coordination of the study and helped to draft the manuscript. DC laboratory director, participated in design of the study and helped to draft the manuscript. VC designed the study, carried out the molecular experiments and helped to draft the manuscript. All authors read and approved the final manuscript.

\section{Author details \\ 1 Biosciences Laboratory, Istituto Scientifico Romagnolo per lo Studio e la Cura dei Tumori (IRST) IRCCS, Via P. Maroncelli 40, 47014 Meldola, Italy. ${ }^{2}$ Unit of Biostatistics and Clinical Trials, Istituto Scientifico Romagnolo per lo Studio e la Cura dei Tumori (IRST) IRCCS, Via P. Maroncelli 40, 47014 Meldola, Italy. ${ }^{3}$ Department of Urology, Morgagni Pierantoni Hospital, Forli, Italy. ${ }^{4}$ Pathol- ogy Unit, Morgagni Pierantoni Hospital, Forlì, Italy. ${ }^{5}$ Department of Urology, Bufalini Hospital, Cesena, Italy. ${ }^{6}$ Department of Medical Oncology, Istituto Scientifico Romagnolo per lo Studio e la Cura dei Tumori (IRST) IRCCS, Via P. Maroncelli 40, 47014 Meldola, Italy. ${ }^{7}$ Dipartimento di Scienze Biomediche, Sperimentali e Cliniche, University of Florence, Florence, Italy.}

\section{Acknowledgements}

We thank Veronica Zanoni for her English revision of the text and Ursula Elbling for editing the manuscript.

\section{Competing interests}

The authors declare that they have no competing interests.

\section{Availability of data and materials}

All data generated or analyzed during this study are included in this published article (and its supplementary information files).

\section{Ethics approval and consent to participate}

The study was approved by the Area Vasta Romagna institutional review board, in accordance with the ethical standards laid down in the 1964 Declaration of Helsinki. Written informed consent was obtained from all patients. 
Received: 20 January 2016 Accepted: 16 August 2016 Published online: 30 August 2016

\section{References}

1. Tao ZQ, Shi AM, Wang KX, Zhang WD. Epidemiology of prostate cancer: current status. Eur Rev Med Pharmacol Sci. 2015;19:805-12.

2. Heijnsdijk EA, der Kinderen A, Wever EM, Draisma G, Roobol MJ, de Koning HJ. Overdetection, overtreatment and costs in prostate-specific antigen screening for prostate cancer. Br J Cancer. 2009;101:1833-8.

3. Heijnsdijk EA, de Carvalho TM, Auvinen A, Zappa M, Nelen V, Kwiatkowski $M$, et al. Cost-effectiveness of prostate cancer screening: a simulation study based on ERSPC data. J Natl Cancer Inst. 2014;107:366.

4. Richiardi L, Fiano V, Vizzini L, De Marco L, Delsedime L, Akre O, et al. Promoter methylation in APC, RUNX3, and GSTP1 and mortality in prostate cancer patients. J Clin Oncol. 2009;27:3161-8.

5. Djavan B, Zlotta AR, Remzi M, Ghawidel K, Bursa B, Hruby S, et al. Total and transition zone prostate volume and age: how do they affect the utility of PSA-based diagnostic parameters for early prostate cancer detection? Urology. 1999;54:846-52.

6. Wang R, Chinnaiyan AM, Dunn RL, Wojno KJ, Wei JT. Rational approach to implementation of prostate cancer antigen 3 into clinical care. Cancer. 2009;115:3879-86.

7. Egger G, Liang G, Aparicio A, Jones PA. Epigenetics in human disease and prospects for epigenetic therapy. Nature. 2004:429:457-63.

8. Esteller M. Epigenetics in cancer. N Engl J Med. 2008;358:1148-59.

9. Andres G, Ashour N, Sanchez-Chapado M, Ropero S, Angulo JC. The study of DNA methylation in urological cancer: present and future. Actas Urol Esp. 2013;37:368-75.

10. Feinberg AP, Ohlsson R, Henikoff S. The epigenetic progenitor origin of human cancer. Nat Rev Genet. 2006;7:21-33.

11. Jerónimo C, Bastian PJ, Bjartell A, Carbone GM, Catto JW, Clark SJ, et al. Epigenetics in prostate cancer: biologic and clinical relevance. Eur Urol. 2011;60:753-66

12. Paziewska A, Dabrowska M, Goryca K, Antoniewicz A, Dobruch J, Mikula M, Jarosz D, et al. DNA methylation status is more reliable than gene expression at detecting cancer in prostate biopsy. Br J Cancer. 2014;12(111):781-9.

13. Cho KH, Choi MJ, Jeong KJ, Kim JJ, Hwang MH, Shin SC, Park CG, et al. A ROS/STAT3/HIF-1a signaling cascade mediates EGF-induced TWIST1 expression and prostate cancer cell invasion. Prostate. 2014;74:528-36.

14. Stone L. Prostate cancer: PTEN loss and PSGR overexpression promote cancer progression. Nat Rev Urol. 2015;12:364.

15. Murria R, Palanca S, de Juan I, Egoavil C, Alenda C, García-Casado Z, et al Methylation of tumor suppressor genes is related with copy number aberrations in breast cancer. Am J Cancer Res. 2014;5:375-85.

16. Castro M, Grau L, Puerta P, Gimenez L, Venditti J, Quadrelli S, et al. Multiplexed methylation profiles of tumor suppressor genes and clinical outcome in lung cancer. J Transl Med. 2010;8:86.

17. Molinari C, Casadio V, Foca F, Zingaretti C, Giannini M, Avanzolini A, et al. Gene methylation in rectal cancer: predictive marker of response to chemoradiotherapy? J Cell Physiol. 2013:228:2343-9.

18. Moelans CB, de Groot JS, Pan X, van der Wall E, van Diest PJ. Clonal intratumor heterogeneity of promoter hypermethylation in breast cancer by MS-MLPA. Mod Pathol. 2014;27:869-74.

19. Garcia-Baquero R, Puerta P, Beltran M, Alvarez-Mujica M, Alvarez-Ossorio $J$, Sanchez-Carbayo M. Methylation of tumor suppressor genes in a novel panel predicts clinical outcome in paraffin-embedded bladder tumors. Tumour Biol. 2014;35:5777-86.

20. Casadio V, Molinari C, Calistri D, Tebaldi M, Gunelli R, Serra L, et al. DNA methylation profiles as predictors of recurrence in non muscle invasive bladder cancer: an MS-MLPA approach. J Exp Clin Cancer Res. 2013;32:94.

21. Hattermann K, Mehdorn HM, Mentlein R, Schultka S, Held-Feindt J. A methylation-specific and SYBR-green-based quantitative polymerase chain reaction technique for O6-methylguanine DNA methyltransferase promoter methylation analysis. Anal Biochem. 2008;377:62-71.
22. Zhu W, Qin W, Hewett JE, Sauter ER. Quantitative evaluation of DNA hypermethylation in malignant and benign breast tissue and fluids. Int J Cancer. 2010:126:474-82

23. Bittner $M$, Meltzer $P$, Chen $Y$, Jiang $Y$, Seftor $E$, Hendrix $M$, et al. Molecular classification of cutaneous malignant melanoma by gene expression profiling. Nature. 2000;406:536-40.

24. Nygren AO, Ameziane N, Duarte HM, Vijzelaar RN, Waisfisz Q, Hess CJ, et al. Methylation-specific MLPA (MS-MLPA): simultaneous detection of CpG methylation and copy number changes of up to 40 sequences. Nucleic Acids Res. 2005;33:e128.

25. Schouten JP, McElgunn CJ, Waaijer R, Zwijnenburg D, Diepvens F, Pals G. Relative quantification of 40 nucleic acid sequences by multiplex ligation-dependent probe amplification. Nucleic Acids Res. 2002;30:e57.

26. Cabello MJ, Grau L, Franco N, Orenes E, Alvarez M, Blanca A, et al. Multiplexed methylation profiles of tumor suppressor genes in bladder cancer. J Mol Diagn. 2011;13:29-40.

27. Yang B, Sun H, Lin W, Hou W, Li H, Zhang L, et al. Evaluation of global DNA hypomethylation in human prostate cancer and prostatic intraepithelial neoplasm tissues by immunohistochemistry. Urol Oncol. 2013;31:628-34.

28. Zelic R, Fiano V, Grasso C, Zugna D, Pettersson A, Gillio-Tos A, et al. Global DNA hypomethylation in prostate cancer development and progression: a systematic review. Prostate Cancer Prostatic Dis. 2015;18:1-12.

29. Woodson K, Hayes R, Wideroff L, Villaruz L, Tangrea J. Hypermethylation of GSTP1, CD44, and E-cadherin genes in prostate cancer among US Blacks and Whites. Prostate. 2003;55:199-205.

30. Van Neste L, Herman JG, Otto G, Bigley JW, Epstein JI, Van Criekinge $\mathrm{W}$. The epigenetic promise for prostate cancer diagnosis. Prostate 2012;72:1248-61.

31. Krop I, Player A, Tablante A, Taylor-Parker M, Lahti-Domenici J, Fukuoka $J$, et al. Frequent $\mathrm{HIN}-1$ promoter methylation and lack of expression in multiple human tumor types. Mol Cancer Res. 2004:2:489-94.

32. Hsu A, Wong CP, Yu Z, Williams DE, Dashwood RH, Ho E. Promoter demethylation of cyclin D2 by sulforaphane in prostate cancer cells. Clin Epigenetics. 2011;3:3.

33. Padar A, Sathyanarayana UG, Suzuki M, Maruyama R, Hsieh JT, Frenkel EP, et al. Inactivation of cyclin D2 gene in prostate cancers by aberrant promoter methylation. Clin Cancer Res. 2003;9:4730-4.

34. Brikun I, Nusskern D, Gillen D, Lynn A, Murtagh D, Feczko J, et al. A pane of DNA methylation markers reveals extensive methylation in histologically benign prostate biopsy cores from cancer patients. Biomark Res. 2014;2:25.

35. Stewart GD, Van Neste L, Delvenne P, Delrée P, Delga A, McNeill SA, et al. Clinical utility of an epigenetic assay to detect occult prostate cancer in histopathologically negative biopsies: results of the MATLOC study. J Urol. 2013;189:1110-6.

36. Partin AW, Van Neste L, Klein EA, Marks LS, Gee JR, Troyer DA, et al. Clinical validation of an epigenetic assay to predict negative histopathological results in repeat prostate biopsies. J Urol. 2014;192:1081-7.

37. Murphy AM, McKiernan JM, Olsson CA. Controversies in prostate cancer screening. J Urol. 2004;172:1822-4.

38. Sunami E, Shinozaki M, Higano CS, Wollman R, Dorff TB, Tucker SJ, et al. Multimarker circulating DNA assay for assessing blood of prostate cancer patients. Clin Chem. 2009:55:559-67.

39. Ahmed $\mathrm{H}$. Promoter methylation in prostate cancer and its application for the early detection of prostate cancer using serum and urine samples. Biomark Cancer. 2010;2010:17-33.

40. Casadio V, Calistri D, Tebaldi M, Bravaccini S, Gunelli R, Martorana G, et al. Urine cell-free DNA integrity as a marker for early bladder cancer diagnosis: preliminary data. Urol Oncol. 2013;31:1744-50.

41. Ellinger J, Muller SC, Stadler TC, Jung A, von Ruecker A, Bastian PJ. The role of cell-free circulating DNA in the diagnosis and prognosis of prostate cancer. Urol Oncol. 2011:29:124-9.

42. Roupret M, Hupertan V, Yates DR, Catto JW, Rehman I, Meuth M, et al. Molecular detection of localized prostate cancer using quantitative methylation-specific PCR on urinary cells obtained following prostate massage. Clin Cancer Res. 2007;13:1720-5. 